\title{
CONSTRUCTION OF HELICAL MAGNETS FOR RHIC
}

\author{
E. Willen $^{\#}$, E. Kelly, M. Anerella, J. Escallier, G. Ganetis, A. Ghosh, R. Gupta ${ }^{+}$, A. Jain, A. Marone, \\ G. Morgan, J. Muratore, A. Prodell, and P. Wanderer, BNL, Upton, NY \\ M. Okamura, RIKEN, Japan
}

\begin{abstract}
Helical magnets are required in RHIC to control proton spin in a program for polarized proton colliding beam experiments. The basic construction unit is a superconducting magnet producing a four tesla dipole field that rotates through 360 degrees in a length of 2.4 meters. These magnets are assembled in groups of four to build four Snakes to control spin in the lattice and eight Rotators to orient spin at two collision points. A collaboration with the Japanese institute RIKEN is providing financial support to carry out the program. After a successful $R \& D$ program to validate the design, the magnets are in the construction phase. The design of the magnets will be reviewed, the construction approach will be described, and test results from the first completed units will be presented.
\end{abstract}

\section{INTRODUCTION}

Previous papers [1-5] have described the parameters needed to control the spin of protons in RHIC. Specifications for the magnets include a large aperture $(100 \mathrm{~mm})$, a dipole field $(4 \mathrm{~T})$ that rotates through $360^{\circ}$ in a distance of $2.4 \mathrm{~m}$, and operation at modest current $(<500 \mathrm{~A})$. By the standards of superconducting accelerator magnets, the field quality requirements are modest (harmonics $<10^{-3}$ of main field). The error allowed on the rotation angle is two degrees. The complete program in RHIC requires 48 of these magnets. A half-length prototype tested in 1997 validated the current design $[1,4]$ and led to the construction program now underway.

\section{DESIGN}

A cross section drawing of the helical magnet is shown in Fig. 1. The coil structure consists of aluminum tubes with slots that are filled with Kapton-insulated superconducting cable (called windings). The tubes are surrounded by a yoke made of single piece, low-carbon steel laminations. Holes near the outer perimeter of the yoke allow for tie rods, warm-up heaters, passage of helium coolant, and bus work for magnet interconnections. The slots rotate along the magnet length but the holes in the yoke do not; these were therefore designed with rotational symmetry in mind. Azimuthal Lorentz forces are contained in the individual slots and, in contrast to the case of $\cos \theta$ dipole

*Work supported by RIKEN and by the US Department of Energy \# email: willen@bnl.gov

+Now at LBNL, Berkeley, CA

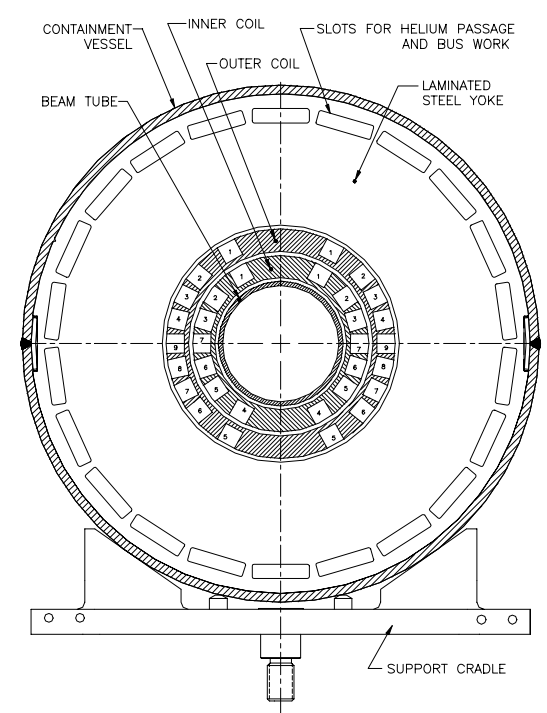

Fig. 1: Cross section of the helical magnet. The yoke OD is 14". The innermost conductor is at an ID of 100 mm. The coil tube outer diameters are 4.945", 6.425".

magnets made with keystoned cable, do not build up on the midplane turns. Along the length of the magnet, the outward Lorentz forces are ultimately contained by the single piece yoke. In the ends [6], the difficult Lorentz force problem is again solved by containing the forces in the individual slots, as shown in Fig. 2. This figure also shows the reliefs machined into the tubes for the leads.

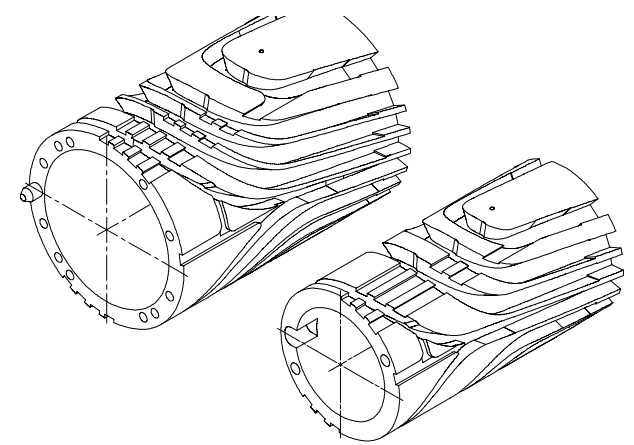

Fig. 2: Drawing of the coil tube ends. End Lorentz forces are contained in the slots.

\section{COILS}

\subsection{Coil Mechanics}

The aluminum tubes are purchased as blanks ready for the machining of the slots. Steps in their manufacture include extrusion, annealing, gun-drilling, honing (ID), and machining (OD). The most critical dimension is the inner surface of the outer tube, where the diameter is specified 
\pm 2 mils. Straightness over the full length is specified \pm 10 mils. Slots are machined into the tubes at BNL using an IGES file generated by AutoCad design software. The machining operation is done on a milling machine using a 0.5 " end mill to cut the 0.560 " wide slot in successive passes, each pass removing a depth of 0.25 " material. A finishing end mill is used to finish the sides and bottom of the slot. All dimensions are measured relative to a keyway or pin at the end of the tube. Errors in slot position are approximately \pm 10 mils or less along the length of the slot. The slot length can be off by up to 20 mils, due primarily to temperature variations and compressive forces during the machining operation.

Before winding, the slots are lined with Kapton for additional electrical insulation of the conductor. Preformed three mil strips secured with two mil 3M Corp. 465 adhesive film are applied along the length of the slot. Fig. 3 is a drawing of the slot showing this insulation. In the ends, the slots are insulated with short $3 / 8$ " wide, one mil thick, $50 \%$ overlapped strips of Kapton backed with silicone adhesive. This is labor intensive but no less

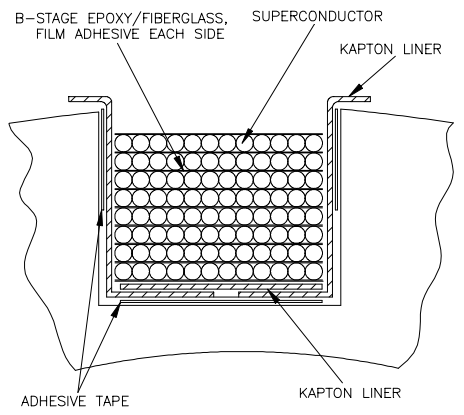

Fig. 3: Cross section of a slot. It is $0.560 "$ wide and 0.512" deep. Each slot has 108 turns except the pole slots, which contain 96 turns. All slots have the same dimensions, which aids the construction.

costly way has been found to apply insulation free of wrinkles and voids in that region. The integrity of the insulation is checked to $4 \mathrm{kV}$ by passing a conductive piece of foam along the full length of each slot. Thin Nomex barriers are used as necessary to prevent damage to the conductor or to the Kapton insulation.

The superconducting cable is placed, either by machine or by hand, without tension into the slots in an ordered array. The width of the slot is such that 12 turns of the cable (each 0.045" diameter including 0.039" cable, 0.004 " Kapton insulation and 0.002" adhesive coating) have on average about one mil space between turns. A layer of B-stage fiberglass/epoxy (either Fiber-Resin Corp. 8600 or Fravillig Materials Corp. epoxy phenolic) with adhesive film (3M 465) on each side is placed between each layer (Fig. 3). This material contains $\sim 40 \%$ by weight of epoxy.

After winding, press plates of 0.125 " thick G10 fiberglass are applied to each slot and secured with ring clamps. These plates are preformed to a helical shape or to the end shape by heating and forcing into an appropriate shallow slot. A wrap of Kevlar 29 (Denier $1000,20 \mathrm{lb}$ tension or $720,10 \mathrm{lb}$ tension) is applied to the tube in a prescribed pattern. The Kevlar stretches and applies pressure on the press plates. In the subsequent curing operation, the stretch in the Kevlar allows the press plates to move radially inward without losing all tension, thereby compressing the windings and removing voids in the slots as the epoxy softens and fills the remaining spaces. Thus, after curing, the cable is held in a strong matrix of fiberglass and resin that can later withstand the Lorentz forces that would cause motion and quenching. Helium is still able to penetrate this package and fill the $\sim 10 \%$ free space inside the Kapton wrap and around the wires of the cable.

As the cable is pressed radially inward during curing, it expands axially because of the helix and, in the ends, because of the curvature of the cylinder. To accommodate this growth, additional axial space (0.030") is allowed in the slot ends. The windings are observed to sometimes fill this space but not to do so consistently. If they do not, the growth is absorbed along the length of the winding. In all cases, any spaces left in the ends after the curing operation are filled with Emerson \& Cummings Corp. Stycast 2850FT epoxy while the structure is being gently vibrated to ensure that all voids are filled. Cut-outs in the press plates provide access to the windings for this filling operation.

After curing to the prescribed recipe, the Kevlar is removed to allow access for the aforementioned filling and the dressing and securing of leads (using Stycast 2850MT epoxy). The tube is then wrapped with a layer of fiberglass cloth to cushion sharp edges that might damage the final Kevlar wrap. A new wrap of Kevlar 29 Denier 1000 is applied with $10 \mathrm{lb}$ tension and a pitch of 0.036". A layer of Tedlar is wrapped over the Kevlar to prevent epoxy of the final wet-wrap of fiberglass and epoxy from penetrating into the Kevlar. This is done to prevent cracking of epoxy bonds due to stress from differential thermal coefficients upon cooldown to cryogenic temperature. Multiple layers of fiberglass cloth and Shell 815V40 epoxy are applied to build a radial thickness sufficient to avoid hollows when the tube is subsequently machined to its finished diameter. The tube is continuously rotated while this epoxy cures.

\subsection{Slot Wiring}

A machine has been built to place the insulated cable into the slots under computer control. The computer follows the same slot description used by the milling machine that cut the slots. The stylus of the machine guides the cable into its proper position and, using ultrasonic energy, melts the adhesive coating on the cable sufficiently to fasten it to the substrate. After each layer is wound, the machine pauses while the operator places the next layer of substrate. The machine has been used to wind a number of slots but, because of materials problems and error 
accumulation in slot position, is not yet ready for routine production. In the meantime, the slots are being wound manually. The wiring is checked by applying high voltage $(1 \mathrm{kV})$ and by measuring resistance.

\section{MAGNET ASSEMBLY}

The two coils are first assembled together, then aligned and pinned in a fixture using machined features on the tube ends. This assembly is then oriented vertically and bolted to a plate that also serves as the primary alignment reference in the magnet. Still in a vertical orientation, stacks of yoke laminations several inches high are lowered around the coils with tooling designed to hold the laminations level so as to avoid binding. Typical clearances between tubes and between tube and yoke are several mils. The straightness of the tubes is critical for this assembly, and the use of aluminum tubes ensures freedom from stresses that might distort the tubes when slots are machined into their walls. After the pre-weighed number of laminations is installed, stainless steel tie bars are inserted through four of the yoke holes and secured with bolts that hold the lamination stack. The yoke length is thus controlled by the machined length of the tie bars. The tie bars and the coils are held on a plate at only the lead end of the coils. The coils, controlled by the aluminum tubes, are therefore free to contract axially inside the yoke upon cooldown. A photograph of a completed magnet (the first production unit) is shown in Figure 4.

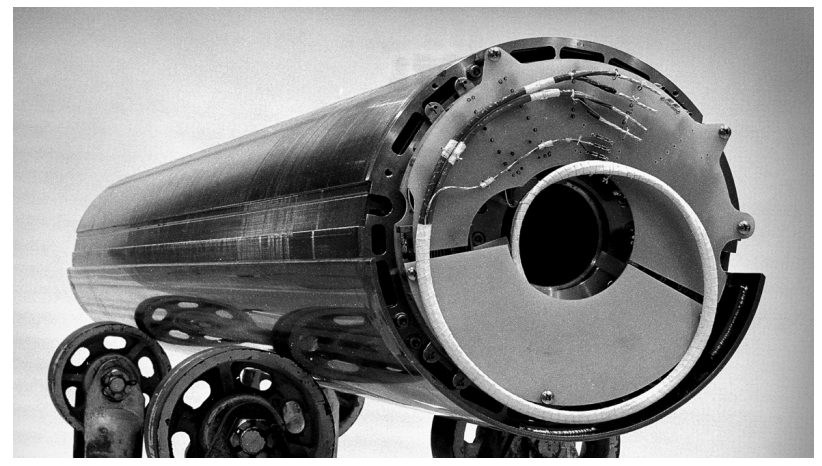

Fig. 4: Photograph of a completed production magnet. It is $2.4 \mathrm{~m}$ long. Electrical connections are under the G10 boards seen at the end of the magnet.

\section{ELECTRICAL CONNECTIONS}

Yoke warmup heaters are installed through four of the yoke slots, then circuit boards are fastened to the lead end of the magnet. Each of the individual windings is connected in a series connection on the circuit boards. A $50 \mathrm{~m} \Omega$ resistor is connected across each of the windings to avoid damage that might occur from energy dissipation in a winding when the magnet quenches. These resistors are made of five folded 12.6" lengths of 16 gauge nichrome wire brazed in parallel into a copper block.
Warm field measurements are made before the resistors are installed.

The room temperature resistance of a winding is typically $20 \Omega$. The average inductance of a winding is $0.3 \mathrm{H}$. The large inductance of the windings and the 50 $\mathrm{m} \Omega$ parallel resistance leads to an indeterminate field in the magnet when it is ramped. This is acceptable because the magnet is designed to be operated only in a DC mode or with very slow $(<1 \mathrm{~A} / \mathrm{s})$ ramp rates. Diodes could be used in series with the resistors if faster ramp rates were needed.

\section{TEST RESULTS}

The first full-length magnet was recently completed and tested at BNL. Quench results at $4.35 \mathrm{~K}$ are shown in Fig. 5. Although some training was necessary, the magnet eventually reached a level well above the

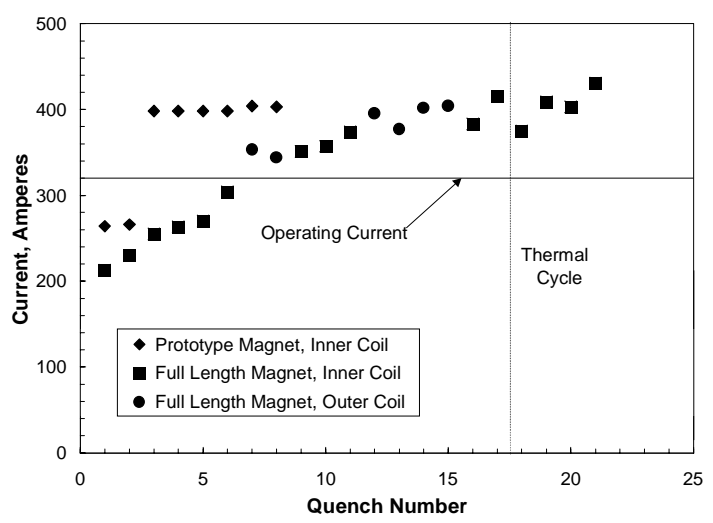

Fig. 5: Quench results for the first full length production magnet, and the earlier prototype magnet.

maximum required operating current. The training may indicate that the windings are not sufficiently filled with epoxy in this first production unit. The test results for the half-length prototype magnet tested in 1997, where no training was observed after several early lead-related quenches, are shown for comparison.

\section{REFERENCES}

[1] E. Willen et al., A Helical Magnet Design for RHIC, Proc. PAC97, Vancouver, BC, May, 1997, p. 3362.

[2] M. Syphers et al., Helical Dipole Magnets for Polarized Protons in RHIC, ibid, p. 3359.

[3] Design Manual: Polarized Proton Collider at RHIC, M. Syphers, Editor, BNL, July, 1998.

[4] M. Okamura et al., Field Calculations and Measurements of a Helical Snake Magnet for RHIC, MT15, Beijing, October, 1997, p. 250.

[5] T. Katayama et al., Field Calculation and Measurement of a Full Length Snake Magnet for RHIC, EPAC, Stockholm, June, 1998, p. 2005.

[6] G. Morgan, End Design of a Helically-Wound Magnet for RHIC, ICEC17, Bournemouth, England, July, 1998, p. 431. 